\title{
Long-term baicalin administration ameliorates metabolic disorders and hepatic steatosis in rats given a high-fat diet
}

\author{
Hong-xia GUO\#, Dai-hua LIU\#, Ying MA, Jin-feng LIU, Ying WANG, Zhi-yan DU, Xin WANG, Jing-kang SHEN, Hong-li PENG* \\ State Key Laboratory of Drug Research, Shanghai Institute of Materia Medica, Chinese Academy of Sciences, Shanghai 201203, \\ China
}

\begin{abstract}
Aim: Baicalin, one of the major flavonoids in Scutellaria baicalensis, possesses antioxidant and anti-inflammatory properties. However, the effects of baicalin on metabolic disorders and hepatic steatosis have not been investigated.

Methods: Body weight was examined in high-fat diet (HFD)-fed rats with or without baicalin treatment. At the end of the experiment, serum biochemical parameters, liver histology and lipid profile were analyzed to assess whether the animals were suffering from metabolic disorders or hepatic steatosis. In the liver, the phosphorylation of AMP activated protein kinase (AMPK) and acetyl-CoA carboxylase (ACC) and the gene expression of some enzymes involved in lipogenesis were examined. The effects of baicalin on the phosphorylation of AMPK and lipid accumulation induced by high glucose in human hepatoma HepG2 cells were also examined.

Results: Baicalin $(80 \mathrm{mg} / \mathrm{kg}$ ) administered ip for 16 weeks suppressed body weight gain in HFD-fed rats. Weight reduction was accompanied by the reduction of visceral fat mass. Baicalin significantly decreased the elevated serum cholesterol, free fatty acid and insulin concentrations caused by the HFD. Baicalin also suppressed systemic inflammation by reducing the serum level of tumor necrosis factor $\alpha$. Baicalin reduced hepatic lipid accumulation, enhanced the phosphorylation of AMPK and ACC and down-regulated genes involved in lipogenesis, including fatty acid synthase and its upstream regulator SREBP-1c. In HepG2 cells, baicalin (5 and $10 \mu \mathrm{mol} / \mathrm{L}$ ) increased the phosphorylation of AMPK and decreased lipid accumulation following the addition of high glucose.

Conclusion: Our study suggests that baicalin might have beneficial effects on the development of hepatic steatosis and obesity-related disorders by targeting the hepatic AMPK.
\end{abstract}

Keywords: baicalin; hepatic steatosis; AMPK; tumor necrosis factor $\alpha$

Acta Pharmacologica Sinica (2009) 30: 1505-1512; doi: 10.1038/aps.2009.150

\section{Introduction}

The excess consumption of fat-enriched food and sedentary lifestyle result in obesity, which is currently a worldwide health problem. A large body of evidence shows that obesity, especially visceral obesity, is associated with an impaired free fatty acid (FFA) metabolism ${ }^{[1]}$. An excessive release of FFA and adipocytokines [such as adiponectin, leptin, and tumor necrosis factor (TNF)- $\alpha$ ] from adipocytes in visceral fat has been suggested to contribute to the development of metabolic syndromes and fatty liver disease, also known as Nonalcoholic Fatty Liver Disease (NAFLD), which is emerging as an obesity-related disorder in obese patients ${ }^{[2-5]}$. Although individuals with NAFLD often live for decades without experiencing

\footnotetext{
\# Both authors contributed equally to this work.

* To whom correspondence should be addressed.

E-mail pengh।@mail.shcnc.ac.cn

Received 2009-05-12 Accepted 2009-09-14
}

clinically significant symptoms of fatty livers, they are unusually susceptible to injury induced by secondary inflammatory stress and may progress to hepatocellular carcinoma ${ }^{[4,6]}$. Since there is currently no effective therapy for NAFLD ${ }^{[7]}$, medications for treating obesity-related fatty liver disorders are urgently needed.

There is an ever-increasing interest in the exploration of natural products as drug candidates ${ }^{[8]}$. Recently, polyphenols have been reported to reduce hyperlipidemia and hyperglycemia, and their effects on lipogenesis have been suggested to be mediated via activation of the AMP-activated protein kinase $(\mathrm{AMPK})^{[9-11]}$. In this relation, AMPK has emerged as an important regulator of lipid metabolism at the cellular and systemic levels, and dysfunction of hepatic AMPK represents a key mechanism for hepatic lipid accumulation and hyperlipidemia with hepatic steatosis in genetically obese rodents ${ }^{[12,13]}$ and in high-fat-fed mice ${ }^{[13]}$ and rats ${ }^{[9,14]}$. Consistent with this observation, transgenic mice expressing constitutively active 
(CA)-AMPKa 1 in liver had reduced levels of white fat and were resistant to high-fat diet-induced obesity ${ }^{[15]}$. AMPK activators, metformin and thiazolidinediones, have been shown to decrease hepatic fat and to improve fatty liver disease, which provides a new therapeutic approach for the treatment of NAFLD $^{[16,17]}$.

Baicalin, a polyphenolic compound, is a major bioactive flavonoid isolated from the radix of Scutellaria baicalensis, which is widely used in traditional Chinese medicine (TCM) to treat inflammation, hypertension, cardiovascular diseases, and bacterial and viral infections ${ }^{[18]}$. Scutellaria baicalensis also shows promise in preventing dyslipidemia and ameliorating hyperglycemia in STZ-induced diabetic rats, both alone and in combination with other herbs ${ }^{[19]}$. Studies with an experimental model demonstrated that extracts of Scutellaria baicalensis significantly decreased the plasma total cholesterol and triglyceride levels of rats fed a high-fat diet ${ }^{[20]}$. Scutellaria baicalensis also reduces plasma triglyceride levels in humans ${ }^{[21]}$, inhibits the progression of atherosclerosis in rabbits with diet-induced hypercholesterolemia ${ }^{[22]}$, and improves lipid profiles in a dietary model of NAFLD ${ }^{[23]}$. However, the molecular basis of pharmacological mechanisms of these hypolipidemic effects is not known. The aim of the present study was to investigate whether baicalin exerts a protective effect in a fat-induced rat model for obesity and fatty liver and whether activation of AMPK plays a role in the action of baicalin. In the present study, we demonstrated that baicalin was able to activate AMPK both in vivo and in cultured cells, leading to a decrease in serum and hepatic lipid levels.

\section{Materials and methods}

\section{Animal experiments}

All animal care and experimental procedures were carried out in accordance with the guidelines of the Laboratory Animal Science Center at the Shanghai Institute of Materia Medica. Male Sprague-Dawley rats (6-8 weeks old) were purchased from SLAC Laboratory Animals Co (Shanghai, China) and maintained in groups under a 12-h/12-h light-dark cycle in a temperature controlled environment $\left(25^{\circ} \mathrm{C}\right)$ with free access to food and water. Animals were fed either a standard laboratory diet (STD, SLAC Laboratory Animals Co) or a high-fat diet (HFD). The STD (by weight) contains $21 \%$ protein, $4.5 \%$ fat, and $52 \%$ carbohydrate. The HFD contains $26.5 \%$ protein, $1 \%$ cholesterol, $0.4 \%$ sodium cholate, $35.4 \%$ saturated fat (lard), and 26.6\% carbohydrate. Both diets were commercially produced (SLAC Laboratory Animals Co, Shanghai, China). After 1 week, rats in the HFD group were further divided into two groups. The control HFD group $(n=7)$ received an intraperitoneal administration of vehicle (sterilized saline) and the baicalin treatment group (HFD+baicalin, $n=8$ ) received an intraperitoneal administration of $80 \mathrm{mg} / \mathrm{kg}$ baicalin in vehicle once per day. The STD group $(n=6)$ also received intraperitoneally administered vehicle. The body weight of individual rats was monitored weekly. After 16 weeks of the treatments and one overnight fasting, rats were anesthetized with chloral hydrate and killed. Serum samples were collected for bio- chemical parameter determination, and tissues were rapidly removed and either frozen immediately in liquid nitrogen or fixed. The visceral fat mass was determined via dissection of epididymal, retroperitoneal, and mesenteric fat deposits.

\section{Cell culture}

Human hepatoma HepG2 cells obtained from the American Type Culture Collection (ATCC) were cultured in MEM medium containing $10 \%$ (vol/vol) fetal bovine serum, 2 $\mathrm{mmol} / \mathrm{L}$ L-glutamine, $1 \mathrm{mmol} / \mathrm{L}$ sodium pyruvate, $100 \mathrm{U} / \mathrm{mL}$ penicillin, $100 \mu \mathrm{g} / \mathrm{mL}$ streptomycin, and $1 \mathrm{mmol} / \mathrm{L}$ sodium pyruvate at $37^{\circ} \mathrm{C}$ in a humidified atmosphere containing $5 \%$ $\mathrm{CO}_{2}$. Cells were grown to $70 \%$ confluence and incubated in serum-free medium overnight before treatments.

\section{Serum biochemical assays}

Serum was separated immediately after blood sampling by centrifugation at $10000 \times g$ for $5 \mathrm{~min}$. Serum concentrations of glucose, total cholesterol, HDL cholesterol, free fatty acids, and triglycerides were determined by enzymatic colorimetric methods using commercially available kits (Shanghai Mind Bioengineering Co, China). Serum LDL cholesterol concentration was calculated by subtracting the HDL cholesterol concentration from the total cholesterol concentration. Serum concentrations of insulin were assessed using a Millipore rat insulin ELISA kit according to the manufacturer's protocol (Millipore, USA). Briefly, the samples were added to wells of a microtiter plate coated by a pre-titered amount of monoclonal anti-rat insulin antibody. Then, biotinylated polyclonal antibodies were added to the captured insulin from samples. After unbound material was washed out of the wells, horseradish peroxidase was added to bind the immobilized biotinylated antibodies, and the enzyme activity was measured spectrophotometrically as increased absorbency at $450 \mathrm{~nm}$, normalized to the absorbency at $590 \mathrm{~nm}$. Serum concentrations of TNF-a were measured using commercially available ELISA kits (PeproTech, USA).

\section{Histological examination}

For analysis of fat accumulation in the liver, the tissue was fixed in $10 \%$ formalin. Frozen sections ( $5 \mu \mathrm{m}$ thick) were prepared using a cryostat, stained with Oil Red O, counterstained with hematoxylin, and analyzed by light microscopy.

\section{Western blot analysis}

Tissues were homogenized or cells were harvested in a lysis buffer $(20 \mathrm{mmol} / \mathrm{L}$ Tris pH 7.5, $150 \mathrm{mmol} / \mathrm{L} \mathrm{NaCl}, 1 \mathrm{mmol} / \mathrm{L}$ EDTA, 1 mmol/L EGTA, 1\% Triton X-100, $2.5 \mathrm{mmol} / \mathrm{L}$ sodium pyrophosphate, $1 \mathrm{mmol} / \mathrm{L} \beta$-glycerolphosphate, $1 \mathrm{mmol} / \mathrm{L}$ $\mathrm{Na}_{3} \mathrm{VO}_{4}, 1 \mu \mathrm{g} / \mathrm{mL}$ leupeptin, $1 \mathrm{mmol} / \mathrm{L}$ PMSF). Samples were sonicated three times for $5 \mathrm{~s}$ with $15 \mathrm{~s}$ breaks between cycles and subsequently centrifuged at $16000 \times \mathrm{g}$ for $60 \mathrm{~min}$ at $4{ }^{\circ} \mathrm{C}$. The protein concentrations of the supernatants were determined with a protein assay kit (Bio-Rad). Equal amounts of total cellular protein were resolved by $10 \%$ SDS-PAGE, transferred onto polyvinylidene difluoride membranes (Amersham 
Biosciences), and then probed with primary antibody followed by secondary antibody conjugated with horseradish peroxidase. The immunocomplexes were visualized via enhanced chemiluminescence (Amersham Biosciences).

\section{Real-time quantitative PCR analysis}

Total RNA was isolated from the liver of each rat using TRIzol reagent (Invitrogen, Carlsbad, CA, USA). The mRNA levels of sterol regulatory element-binding protein (SREBP)-1c and fatty acid synthase (FAS) were assessed by real-time quantitative RT-PCR. PCR reactions were carried out using the DNA Engine Opticon PCR cycler 2 (MJ Research Inc, Waltham, MA, USA) and the Primescript ${ }^{\mathrm{TM}}$ RT reagent Kit (TaKaRa). Primers for SREBP-1C, FAS, and $\beta$-actin were as follows: SREBP-1c (forward, 5' -AGCTCACGGTACCAGCAAT-3'; reverse, 5'-GTAGGAAGACCCTCCTCATA-3'), FAS (forward, 5'-ATGGGAAGGTGTCTGTGCACAT-3'; reverse, 5'-TGTGGATGATGTTGATGATA-3'), $\beta$-actin (forward, $5^{\prime}$-TATGAGGGTTACGCGCTCCC-3'; reverse, 5'-TCTTTAATGTCACGCACGATTTCC-3'). The conditions for PCR were as follows: denaturation at $95^{\circ} \mathrm{C}$ for $5 \mathrm{~s}$ (10 s in the first cycle), annealing at $60^{\circ} \mathrm{C}$ for $30 \mathrm{~s}$, and extension at $72{ }^{\circ} \mathrm{C}$ for $10 \mathrm{~min}$. A melting curve from $55^{\circ} \mathrm{C}$ to $95{ }^{\circ} \mathrm{C}$ was generated at the end of every PCR reaction. PCR reactions for each sample were conducted in triplicate. Relative quantities of mRNA were calculated from $\mathrm{Ct}$ values by the comparative $\mathrm{Ct}$ method using $\beta$-actin as an internal reference.

\section{Determination of intracellular lipid content}

Liver tissues were homogenized (1:20, wt/vol) with hexane and isopropanol (3:2, vol/vol). The mixture was vortexed vigorously and allowed to separate into two phases at room temperature. An aliquot of the organic phase was evaporated at $37^{\circ} \mathrm{C}$ under a vacuum until dry. Hepatic total cholesterol and free fatty acid concentrations in the extracts were determined using the same enzymatic kits that were applied for serum analyses and normalized to liver weight. The results are expressed as milligrams of lipid per gram of tissue weight.

For cell culture studies, HepG2 cells were grown in 100-mm culture dishes and incubated in serum free medium with a normal $(5.5 \mathrm{mmol} / \mathrm{L})$ or high $(30 \mathrm{mmol} / \mathrm{L})$ glucose concentration in the absence or presence of baicalin for $24 \mathrm{~h}$. After treatment, cells were washed twice with cold PBS and subjected to lipid extraction using a modified method ${ }^{[24]}$. In brief, $2 \mathrm{~mL}$ of hexane and isopropanol (3:2, vol/vol) was added to cell layers and maintained for $1 \mathrm{~h}$ at room temperature. The solvent was then transferred to a glass tube and dried under vacuum. The lipids were dissolved in isopropanol and the triglyceride and total cholesterol contents were determined using the enzymatic kits described above. The cellular protein content present in the residue obtained after lipid extraction was dissolved in $\mathrm{NaOH}(0.1 \mathrm{~mol} / \mathrm{L})$ and determined with a protein assay kit (Bio-Rad). Data are expressed as $\mu \mathrm{g}$ lipids $/ \mathrm{mg}$ protein.

\section{Chemicals}

Baicalin (HPLC content 98\%) was purchased from Nanjing
Chongyuan Bio-tech Co Ltd (Nanjing, China). Anti-phosphoAMPKa (Thr172) and anti-AMPKa antibodies were purchased from Cell Signaling Technology. Phospho-ACC (Ser79) antibody was purchased from Upstate Biotechnology. Anti-ACC polyclonal antibody was purchased from Millipore (USA). $\beta$-actin antibody was purchased from Lab Vision Corporation (Fremont, CA). Anti-mouse and anti-rabbit antibodies conjugated to horseradish peroxidase were obtained from Kirkegaard \& Perry Laboratories (Gaithersburg, MD). Minimum Essential Medium (MEM), fetal bovine serum, penicillinstreptomycin solution, sodium pyruvate and TRIzol were purchased from Invitrogen. AICA-Riboside and protease inhibitor cocktail set I were purchased from Calbiochem. All other reagents used were of analytical grade.

\section{Statistical analysis}

All data are presented as means \pm SEM. Student's $t$ test (unpaired) was used to determine the statistical significance $(P<0.05)$ of all obtained data.

\section{Results}

Baicalin treatment reduced body weight gain and body fat mass of the HFD-fed rats

We used a rat model of the HFD feeding for obesity and hepatic steatosis. We first evaluated the chronic effects of baicalin on body weight in this animal model. As shown in Figure 1, rats fed the HFD showed a rapid weight gain and a final body weight $(720.2 \pm 17.4, n=7)$ that was significantly higher than the body weight of rats fed the STD $(629.4 \pm 12.5$ $\mathrm{g}, n=6, P<0.05$; Figure 1A). Daily administration of baicalin $(80 \mathrm{mg} / \mathrm{kg})$ to rats on the HFD for 16 weeks produced a body weight $(632.5 \pm 21.2 \mathrm{~g}, n=8)$ that was markedly lower than that of vehicle-treated HFD control rats $(P<0.05)$ and comparable to that of STD-fed rats (Figure 1A). Similarly, the HFD-fed rats showed a significant increase in total visceral fat mass, including epididymal and perirenal fat, at the end of the experiment $(P<0.05$, Figure 1B). Baicalin treatment also reduced the visceral and epididymal fat mass of HFD-fed rats $(P<0.05$, Figure 1B). Overall food intake did not differ among groups in this long-term experiment (data not shown).

\section{Effect of baicalin on serum biochemical parameters in rats fed the HFD}

At the end of the 16 week study, the HFD significantly increased the fasting serum concentrations of total cholesterol, LDL-cholesterol, NEFA, glucose, insulin, and TNFa compared with the STD. Long-term treatment with baicalin markedly reduced the increase in total cholesterol, LDL-cholesterol, NEFA, insulin and TNFa levels caused by HFD feeding to levels similar to those found in the STD group. Baicalin treatment also significantly reduced the fasting serum glucose levels in rats fed the HFD (Table 1).

Baicalin reduced liver mass and hepatic lipids level in the HFDfed rats

Chronic HFD exposure causes fat accumulation in the liver, 

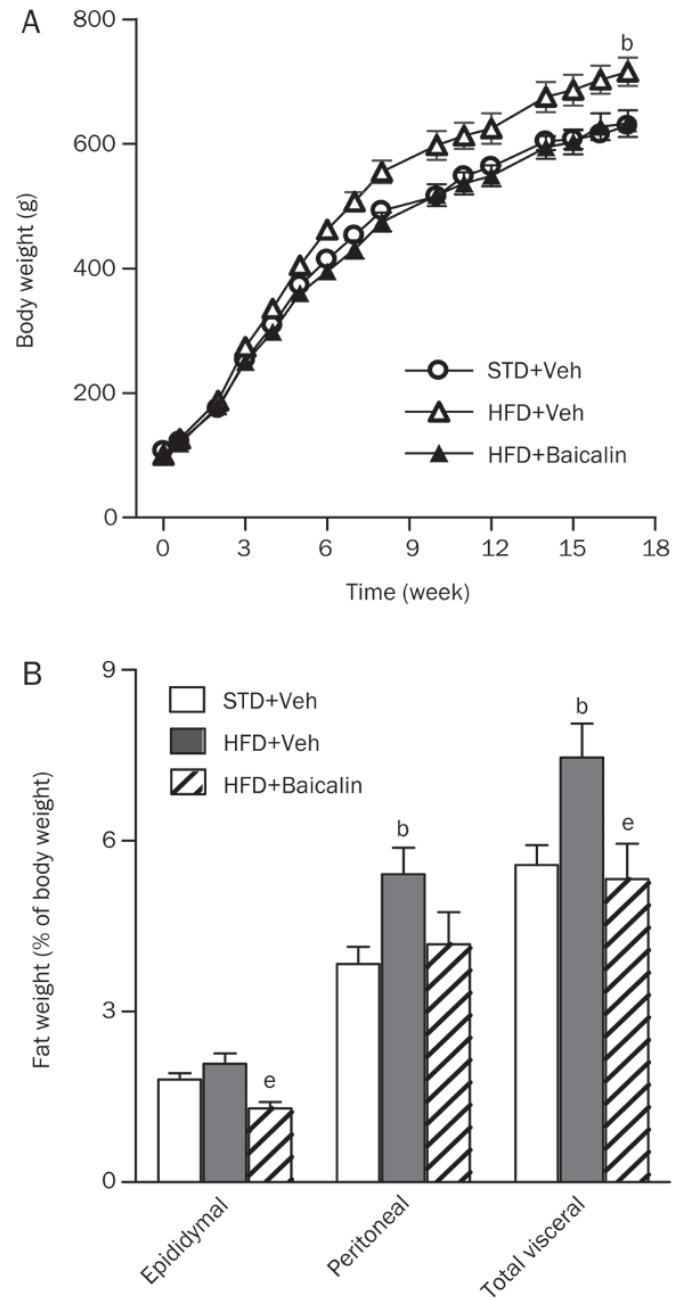

Figure 1. Long-term baicalin administration prevents obesity in rats fed a high-fat diet (HFD). Male Sprague-Dawley rats were fed a standard diet and treated with vehicle (STD+Veh), fed a HFD and treated with vehicle (HFD+Veh), or fed a HFD and treated with baicalin (HFD+baicalin) at a dose of $80 \mathrm{mg} / \mathrm{kg}$ ip daily for 16 weeks. (A) Body weight curves for STD+Veh, HFD+Veh, and HFD+baicalin groups. (B) Fat-to-body weight ratio at the end of the 16-week study. Values are given as means \pm SEM $(n=6-8$ rats per group). ${ }^{\mathrm{b}} P<0.05$ vs STD+Veh; ${ }^{\mathrm{e}} P<0.05$ vs HFD+Veh.

leading to hepatic steatosis. We therefore investigated hepatic accumulation of lipids by measuring lipid contents in the liver and by Oil-red O staining of frozen liver sections. The HFDfed rats have significantly increased liver size (relative to body weight) and liver total cholesterol and NEFA concentrations compared with the STD-fed rats (Figure 2B-2D). Administration of baicalin significantly decreased liver size (Figure $2 B)$ and attenuated fat accumulation in the livers of rats fed the HFD $(P<0.05$, Figure $2 \mathrm{C}$ and $2 \mathrm{D})$. Moreover, histological examination confirmed this effect and showed that the massive hepatic accumulation of lipids in the HFD-fed rats was largely reduced by baicalin treatment (Figure 2A).
Table 1. Biochemical parameters of serum after 16-week treatment with baicalin $\left(80 \mathrm{mg} \cdot \mathrm{kg}^{-1} \cdot \mathrm{d}^{-1} \mathrm{ip}\right)$ in rats fed a HFD. ${ }^{\mathrm{b}} P<0.05$ vs STD+Veh; ${ }^{e} P<0.05$ vs HFD+Veh.

\begin{tabular}{lccc}
\hline & STD+Veh & HFD+Veh & HFD+Baicalin \\
\hline Total cholesterol (mg/dL) & $66.0 \pm 4.5$ & $118.5 \pm 16.5^{\mathrm{b}}$ & $67.6 \pm 5.2^{\mathrm{e}}$ \\
LDL cholesterol (mg/dL) & $10.8 \pm 0.9$ & $16.7 \pm 1.7^{\mathrm{b}}$ & $8.35 \pm 2.41^{\mathrm{e}}$ \\
Triglyceride $(\mathrm{mg} / \mathrm{dL})$ & $109.7 \pm 8.7$ & $90.2 \pm 6.3$ & $89.4 \pm 7.3$ \\
NEFA $(\mathrm{mmol} / \mathrm{L})$ & $1.13 \pm 0.12$ & $3.32 \pm 0.52^{\mathrm{b}}$ & $0.66 \pm 0.17^{\mathrm{e}}$ \\
Glucose $(\mathrm{mg} / \mathrm{dL})$ & $111.1 \pm 5.3$ & $174.2 \pm 5.4^{\mathrm{b}}$ & $145.6 \pm 12.7^{\mathrm{e}}$ \\
Insulin $(\mathrm{ng} / \mathrm{mL})$ & $0.842 \pm 0.071$ & $1.443 \pm 0.169^{\mathrm{b}}$ & $0.796 \pm 0.131^{\mathrm{e}}$ \\
TNF $\alpha(\mathrm{pg} / \mathrm{mL})$ & $320.1 \pm 13.7$ & $808.5 \pm 109.8^{\mathrm{b}}$ & $479.8 \pm 23.3^{\mathrm{e}}$ \\
\hline
\end{tabular}

Values are means \pm SEM. STD+Veh, rats fed a standard diet and received an intraperitoneal administration of vehicle; HFD+Veh, rats fed a highfat diet and received an intraperitoneal administration of vehicle; HFD+ baicalin, rats fed a high-fat diet received an intraperitoneal administration of $80 \mathrm{mg} / \mathrm{kg}$ baicalin once a day; NEFA, nonesterified fatty acids. Means in a row with different superscripts differ significantly.

Baicalin increases AMPK and ACC phosphorylation in the liver of HFD-fed rats

Having established that baicalin had a clear effect on both circulating and hepatic lipid levels, we wanted to assess the possible mechanisms responsible for this effect. AMPK is a metabolic master switch that responds to changes in cellular energy status $^{[25]}$ and has been suggested to play a crucial role in regulating fat metabolism in the liver. AMPK activators, including metformin and thiazolidinediones, prevent the development of hepatic steatosis ${ }^{[26]}$. Therefore, we examined the effect of baicalin on AMPK activation in the liver of HFD-fed rats. As the activity of AMPK correlates strongly with phosphorylation at Thr172 (p-AMPKa), we assessed the activation of AMPK by determining the phosphorylation status of AMPKa and its primary downstream target, acetyl-CoA carboxylase (ACC), using immunoblots with phospho-specific antibodies. As expected ${ }^{[13]}$, the HFD suppressed hepatic AMPKa phosphorylation (activation) and ACC phosphorylation (inactivation) (Figure $3 \mathrm{~A}$ and $3 \mathrm{~B}$ ). Interestingly, baicalin induced a significant recovery of phosphorylation of AMPKa and ACC in liver depressed by the HFD (Figure 3A and 3B).

\section{Hepatic SREBP-1c and FAS mRNA expression}

SREBP-1c is a critical transcriptional factor that is known to regulate the expression of lipogenic enzymes in the liver ${ }^{[27]}$ and is negatively regulated by $\mathrm{AMPK}^{[16]}$. The effects of baicalin treatment on the gene expression of SREBP-1c and its target, FAS, in the liver were examined by quantitative real-time PCR analysis. As shown in Figure 4, when fed a HFD for 16 weeks, rats showed a modest increase in SREBP-1c mRNA levels relative to STD-fed animals. In rats treated with baicalin, however, the levels of liver SREBP-1c mRNA were significantly reduced compared with those of HFD-fed rats (Figure 4). The levels of FAS mRNA were not elevated by HFD feeding 
A

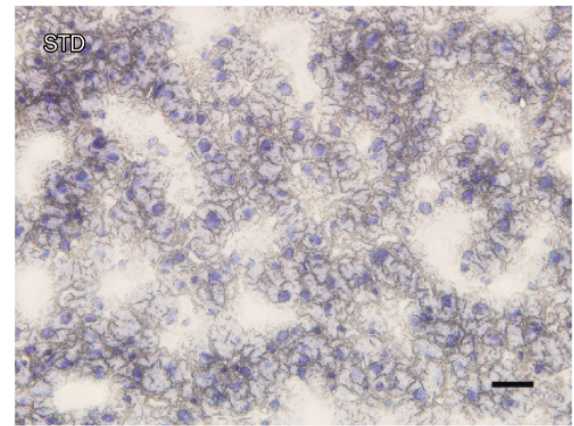

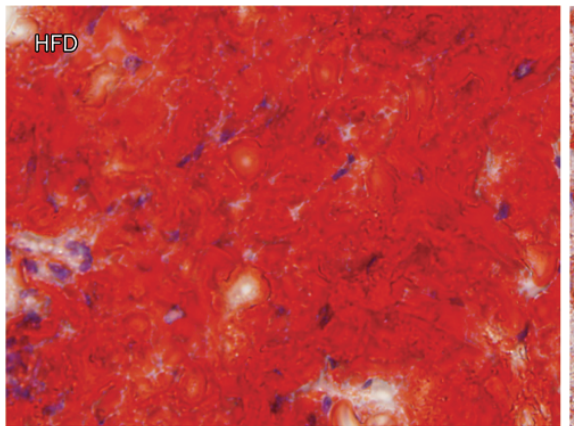

C

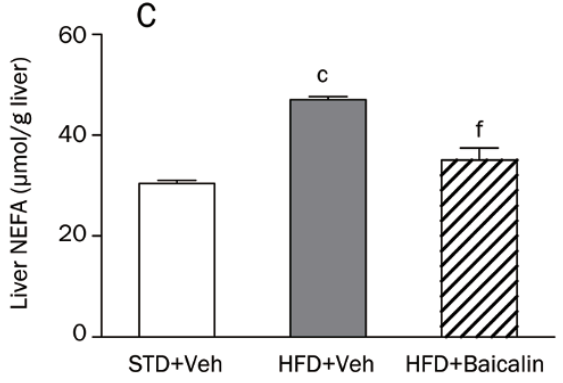

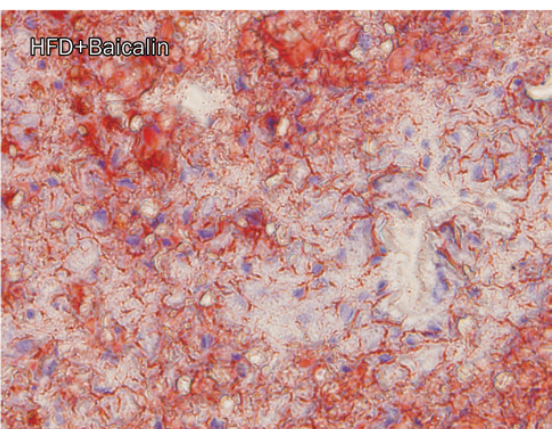

D

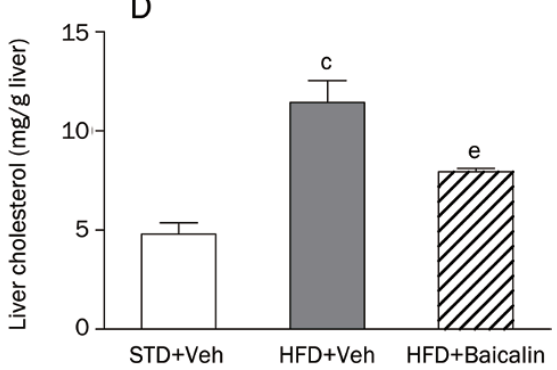

Figure 2. Long-term baicalin administration attenuates fat accumulation in the livers of rats fed a high-fat diet (HFD). Sprague-Dawley rats were fed a standard diet and treated with vehicle (STD+Veh), fed a HFD and treated with vehicle (HFD+Veh), or fed a HFD and treated with baicalin (HFD+baicalin) at a dose of $80 \mathrm{mg} / \mathrm{kg}$ ip daily for 16 weeks. (A) Hepatic histology of a representative rat from each treatment group. Liver sections were fixed in formalin, embedded in paraffin, and stained with Oil Red $\mathrm{O}$ and hematoxylin (original magnification, $\times 400$ ). (B) Liver-to-body weight ratio of STD+Veh (open bars), HFD+Veh (solid bars), and HFD+baicalin (hatched bars). The concentrations of liver total cholesterol (C) and nonesterified fatty acids (NEFA, D) were measured as described under Materials and Methods. Scale bars indicate $200 \mu \mathrm{m}$. Values are given as means \pm SEM ( $n=6-8$ rats per group). ${ }^{\mathrm{c}} P<0.01$ vs STD+Veh; ${ }^{\mathrm{e}} P<0.05,{ }^{\mathrm{f}} P<0.01$ vs HFD+Veh.
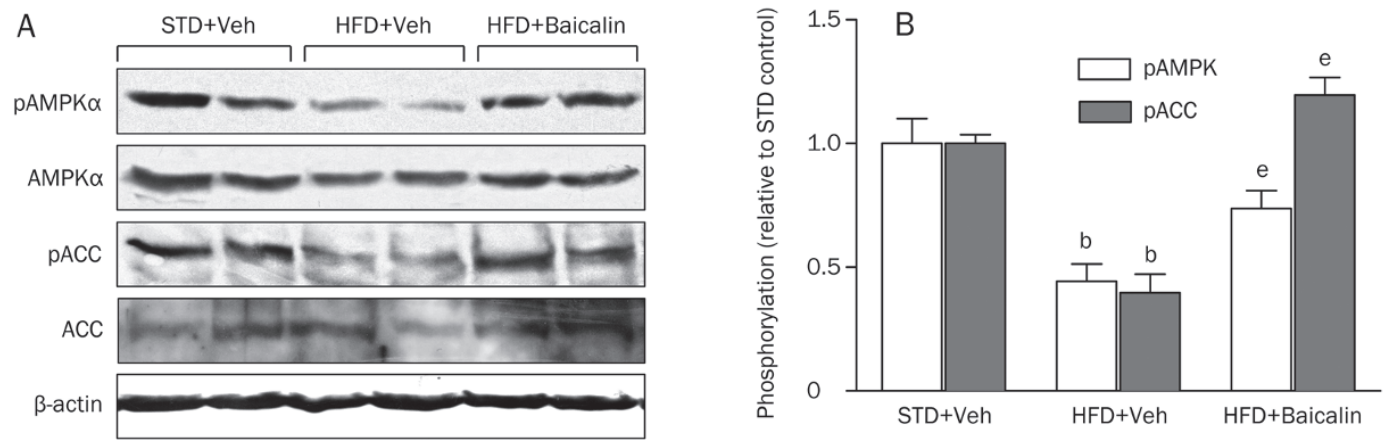

Figure 3. Long-term baicalin administration stimulates phosphorylation of AMPK and ACC in the livers of rats fed a high-fat diet (HFD). Male SpragueDawley rats were fed a standard diet and treated with vehicle (STD+Veh), fed a HFD and treated with vehicle (HFD+Veh) or with baicalin (HFD+baicalin) at a dose of $80 \mathrm{mg} / \mathrm{kg}$ ip daily for 16 weeks. Liver protein extract $(50 \mu \mathrm{g})$ was subjected to Western blot analysis for AMPK $\alpha$ and its downstream target, ACC, using antibodies specific for AMPK $\alpha$ phosphorylated at Thr172 (pAMPK $\alpha$ ) and ACC phosphorylated at Ser79 (pACC). (A) Representative immunoblots for pAMPK $\alpha$, PACC, and expression of total AMPK $\alpha$ and ACC and $\beta$-actin in the livers of two rats in each group. (B) Densitometric analysis of pAMPK $\alpha$ and pACC protein levels. The histogram represents the mean of the density normalized to the corresponding STD control \pm SEM. $n=3$ rats in each group. ${ }^{\mathrm{b}} \mathrm{P}<0.05$ vs $\mathrm{STD}+\mathrm{Veh} ;{ }^{\mathrm{e}} \mathrm{P}<0.05$ vs HFD+Veh.

but were significantly decreased in rats treated with baicalin (Figure 4).

Baicalin activates AMPK and inhibits lipid accumulation in HepG2 cells

The conclusion that baicalin is able to activate AMPK was strengthened by experiments in cultured HepG2 cells. As shown in Figure 5A, incubating HepG2 cells with baicalin at 5 $\mu \mathrm{mol} / \mathrm{L}$ and $10 \mu \mathrm{mol} / \mathrm{L}$ for $1 \mathrm{~h}$ activated AMPK (ie, increased the expression of phospho-AMPKa). AICAR, an AMP analogue and known AMPK activator, was used as a positive control. 


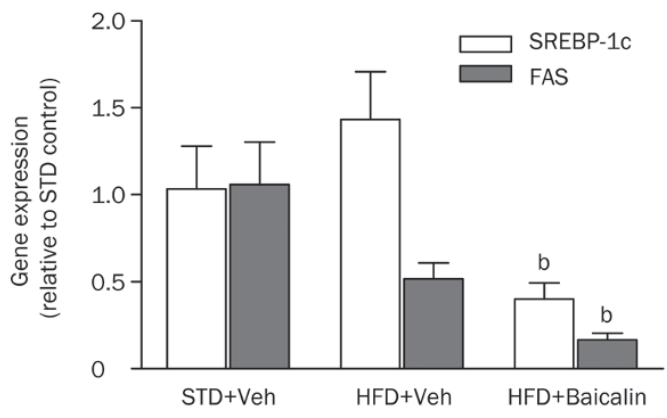

Figure 4. Long-term baicalin administration downregulates the gene expression of SREBP-1C and fatty acid synthase (FAS) in the livers of rats fed a high-fat diet (HFD). Male Sprague-Dawley rats were fed a standard diet and treated with vehicle (STD+Veh) or fed a HFD and treated with vehicle (HFD+Veh) or baicalin (HFD+baicalin) at a dose of $80 \mathrm{mg} / \mathrm{kg}$ ip once a day for 16 weeks. The expression of SREBP-1c and FAS mRNAs in the liver was assayed by real-time quantitative PCR. Each bar represents the mean \pm SEM of RT-PCR analyses with the RNA samples from liver tissues of three to eight animals. All expression levels were normalized to the $\beta$-actin expression in the same PCR reaction. The ratio of mean expression in the STD liver was used as a control (1-fold). ${ }^{b} P<0.05$ vs respective HFD+Veh.
HepG2 cells exposed to high glucose develop insulin resistance due to decreased phosphorylation of AMPK and increased cellular lipids ${ }^{[28]}$. To test whether activation of AMPK by baicalin could prevent the lipid accumulation caused by a high glucose concentration, HepG2 cells were incubated with either normal $(5.5 \mathrm{mmol} / \mathrm{L})$ or high $(30$ $\mathrm{mmol} / \mathrm{L})$ glucose in the absence or presence of baicalin (5 $\mu \mathrm{mol} / \mathrm{L}$ or $10 \mu \mathrm{mol} / \mathrm{L}$ ) for $24 \mathrm{~h}$. As shown in Figure 5, baicalin treatment was still able to induce AMPKa phosphorylation (activation) in cells exposed to elevated glucose (Figure $5 \mathrm{~A})$. Baicalin treatment also reduced the elevated intracellular triglyceride (Figure 5B) and total cholesterol levels induced by high glucose concentrations (Figure 5C).

\section{Discussion}

In the present study, we tested the potential effects of baicalin on HFD-induced metabolic disorders and hepatic steatosis. We used an experimental model of HFD feeding and showed that supplying a HFD to rats for 16 weeks produces a dyslipidemic profile, elevates serum fasting glucose, insulin and TNFa concentrations, and results in significant body weight gain and hepatic steatosis. Treatment with baicalin $(80$ $\mathrm{mg} / \mathrm{kg}$ ), however, ameliorated the dyslipidemia and hepatic lipid accumulation, improved hepatic steatosis, and reduced

A

\begin{tabular}{|c|c|c|c|c|c|c|c|c|}
\hline \multirow{4}{*}{$\begin{array}{r}\text { AICAR }(\mathrm{mmol} / \mathrm{L}) \\
\text { Baicalin }(\mu \mathrm{mol} / \mathrm{L}) \\
\text { Time }(\mathrm{h})\end{array}$} & \multicolumn{4}{|c|}{$N G$} & \multicolumn{4}{|c|}{ HG } \\
\hline & - & 500 & - & - & - & 500 & - & - \\
\hline & - & - & 5 & 10 & - & - & 5 & 10 \\
\hline & - & 4 & 4 & 4 & - & 24 & 24 & 24 \\
\hline pAMPK $\alpha$ & & - & & $\longrightarrow$ & 7 & 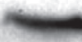 & $\operatorname{lin}$ & N \\
\hline AMPK $\alpha$ & $=$ & & & $=$ & $=$ & & & \\
\hline
\end{tabular}
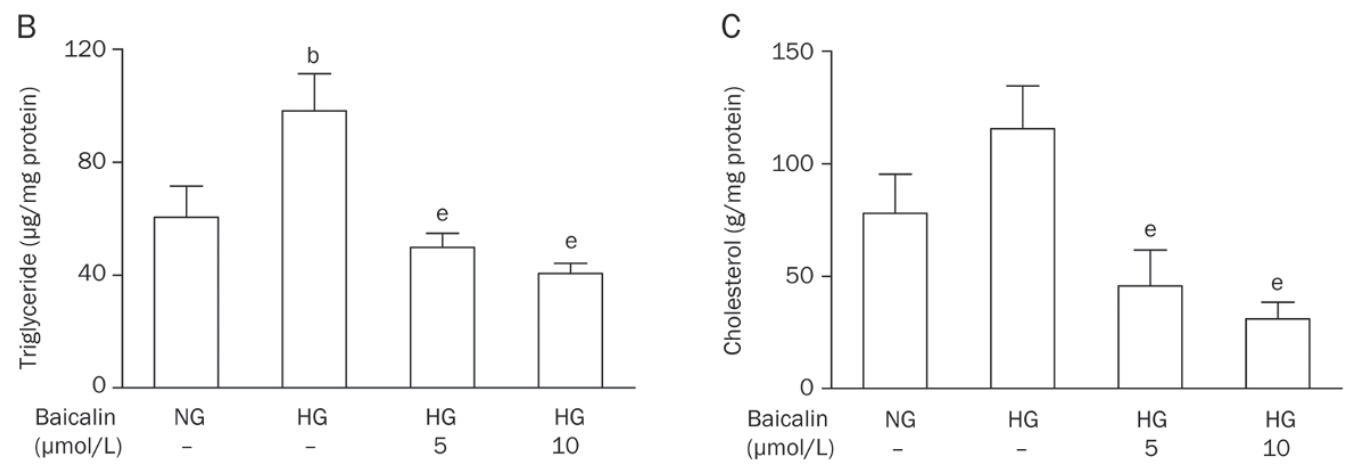

Figure 5. Baicalin stimulates the phosphorylation of AMPK and decreases the lipid accumulation caused by high glucose concentration in HepG2 cells. (A) Representative immunoblots showing the effects of baicalin on the activation of AMPK. HepG2 cells were grown in 100-mm culture dishes until confluence. Cells were maintained in serum-free MEM medium overnight and treated with baicalin ( 5 and $10 \mu \mathrm{mol} / \mathrm{L}$ ) in MEM containing either normal glucose $(5.5 \mathrm{mmol} / \mathrm{L}, \mathrm{NG}$ ) or high glucose ( $30 \mathrm{mmol} / \mathrm{L}, \mathrm{HG}$ ) for the indicated times. AMPK $\alpha$ phosphorylation (pAMPK) and total AMPK $\alpha$ were detected by western blot analysis as described under "Materials and Methods." AICAR was used as a positive control. Immunoblots are representative of three individual experiments. (B) and (C) Baicalin reduced the increase in lipid accumulation induced by HG in HepG2 cells. Cells were cultured in serum-free MEM overnight and incubated in MEM containing HG in the absence or presence of baicalin ( 5 and $10 \mu \mathrm{mol} / \mathrm{L}$ ) for an additional $24 \mathrm{~h}$. Cells in MEM containing NG in the absence of baicalin was used as the control. The data are presented as the means \pm SEM of at least five experiments. ${ }^{b} P<0.05$ vs normal glucose control; ${ }^{\mathrm{e}} \mathrm{P}<0.05$ vs high-glucose untreated control. 
body weight gain accompanied by a significantly decreased visceral fat mass in HFD-fed rats. Baicalin treatment also significantly decreased the elevated serum TNFa and insulin levels caused by HFD feeding. In addition, baicalin enhanced the phosphorylation of AMPK and ACC and suppressed the expression of SREBP-1c and FAS mRNA in the liver. In cultured HepG2 cells, baicalin also increased the levels of active (phosphorylated) AMPKa and decreased lipid accumulation following a rise in glucose level. Our findings are the first to suggest that baicalin might have beneficial effects on obesityrelated disorders and hepatic steatosis by targeting the hepatic AMPK pathway.

Baicalin, a principal active flavonoid of Scutellaria baicalensis, possesses antioxidant and anti-inflammatory properties that have been studied in relation to cardiovascular disease, agerelated changes in physiology, and maintenance of brain function after heatstroke in animal models ${ }^{[2,30]}$. Scutellaria baicalensis is one of the most popular multipurpose herbs used in traditional Chinese medicine. Although Scutellaria baicalensis, both alone and in combination with other herbs, has been used for the clinical treatment of hyperlipidemia and liver diseases for some time ${ }^{[21,31]}$, information about which components in Scutellaria baicalensis might cause these hypolipidemic effects is scarce. Furthermore, the molecular mechanisms through which Scutellaria baicalensis acts are unknown. Recently, beneficial effects on obesity, diabetes and fatty liver have been reported for various flavonoids, which are thought to act through AMPK ${ }^{[9-11]}$. Therefore, the bioactive flavonoids such as baicalin in Scutellaria baicalensis are of particular interest.

The AMPK complex is an evolutionally conserved serine/ threonine heterotrimeric kinase complex that consists of $\alpha-, \beta-$ and $\gamma$-subunits and acts as a "master switch" for lipid metabolism (reviewed $\mathrm{in}^{[25]}$ ). Its activity is abnormal under conditions of deregulated energy balance, such as obesity, diabetes and fatty liver ${ }^{[32]}$. The activation of hepatic AMPK (through phosphorylation of Thr172 of its a-subunit) switches off fatty acid and cholesterol synthesis through two downstream pathways: acutely via increased phosphorylation of ACC and 3-hydroxy3-methylglutaryl (HMG) CoA reductase and chronically via decreased transcription of SREBP-1c and its target genes (eg, FAS) ${ }^{[15,25]}$. Two major classes of insulin-sensitizing drug, the biguanides and the thiazolidinediones, appear to exert their therapeutic effects by activating AMPK. This suggests that activators of AMPK may be useful for treatment of certain metabolic disorders, type 2 diabetes, and obesity ${ }^{[33]}$.

Concomitant with the substantially attenuated fat accumulation in the serum and livers of rats fed a HFD, long-term administration of baicalin enhanced hepatic AMPK activation by promoting AMPK phosphorylation and inhibiting ACC, which is also associated with AMPK activation ${ }^{[34]}$. Long-term administration of baicalin also down-regulated the expression of mRNAs encoding several genes involved in lipid metabolism, including SREBP-1c and FAS in the liver. Activation of AMPK reduces the expression of proteins involved in lipogenesis, including the upstream regulator SREBP ${ }^{[16]}$. These in vivo observations were further supported by experiments in HepG2 cells, in which baicalin treatment led to AMPK activation and decreased lipid accumulation following the addition of high glucose. Thus, AMPK activation appears to play an important role in the effects of baicalin on hepatic steatosis and obesity. The details of the basis for baicalin-mediated activation of AMPK remain unknown.

Several lines of evidence demonstrate that exposure to a HFD and diet-induced obesity lead to overproduction of proinflammatory cytokines such as TNF-a and excessive release of FFA, which are both believed to be critical in the progression of NAFLD ${ }^{[35,36]}$, as well as for the development of insulin resistance ${ }^{[36]}$. Indeed, our data showed that rats fed a HFD for 16 weeks exhibited a significant increase in serum TNFa. In association with this systemic inflammatory stress, prominent hepatic steatosis and a profound increase in serum glucose and insulin levels were shown in HFD-fed rats. Baicalin, which has anti-inflammatory properties, has been reported to inhibit TNF-a overproduction during lipopolysaccharide-induced liver injury in mice ${ }^{[37]}$ and cerebrovascular dysfunction in rats ${ }^{[29]}$. Consistent with these reports, our data also showed that long-term baicalin administration markedly decreased serum TNFa and lowered both serum and hepatic FFA levels compared with HFD-fed rats that did not receive baicalin. Baicalin treatment also improved insulin sensitivity in HFD-fed rats, as demonstrated by a significant reduction in serum insulin and a modest decrease in serum glucose levels. Thus, suppression of TNF-a by baicalin may also contribute to its protective effects against the development of hepatic steatosis caused by HFD feeding.

In conclusion, our data revealed that baicalin treatment protected against the development of hepatic steatosis and obesity induced by a long-term high-fat diet; this protective effect is associated mainly with a significant enhancement of hepatic AMPK activation. In addition, baicalin treatment suppressed systemic inflammatory stress by reducing serum TNF-a levels and ameliorated insulin resistance. Thus, the present study provides a strong rationale for further evaluation of the potential therapeutic role of baicalin in treating obesity-related fatty liver disease.

\section{Acknowledgments}

We thank Dr C AALKJAER for critically reading the manuscript. This study was supported by grants from the National Natural Science Foundation of China (№ 30672467) and the Ministry of Science and Technology (№ 2007AA02Z308).

\section{Author contribution}

Hong-xia GUO and Dai-hua LIU designed and performed the research. Ying MA performed real time quantitative PCR analysis. Jin-feng LIU participated in part of the experiments. Ying WANG and Zhi-yan DU helped with animal experiments. Xin WANG helped with intracellular lipid analysis. Jing-kang SHEN provide consultation. Hong-li PENG wrote the paper. 


\section{References}

1 Boden G. Obesity and free fatty acids. Endocrinol Metab Clin North Am 2008; 37: 635-46.

2 Festi D, Colecchia A, Sacco T, Bondi M, Roda E, Marchesini G. Hepatic steatosis in obese patients: clinical aspects and prognostic significance. Obes Rev 2004; 5: 27-42.

3 Akbar DH, Kawther AH. Non-alcoholic fatty liver disease and metabolic syndrome: what we know and what we don't know. Med Sci Monit 2006; 12: RA23-RA26.

4 Clark JM, Diehl AM. Nonalcoholic fatty liver disease: an underrecognized cause of cryptogenic cirrhosis. JAMA 2003; 289: 3000-4.

5 Wieckowska A, McCullough AJ, Feldstein AE. Noninvasive diagnosis and monitoring of nonalcoholic steatohepatitis: present and future. Hepatology 2007; 46: 582-9.

6 Adams LA, Angulo P, Lindor KD. Nonalcoholic fatty liver disease. CMAJ 2005; 172: 899-905.

7 Younossi ZM. Review article: current management of non-alcoholic fatty liver disease and non-alcoholic steatohepatitis. Aliment Pharmacol Ther 2008; 28: 2-12.

8 Marcaurelle LA, Johannes CW. Application of natural product-inspired diversity-oriented synthesis to drug discovery. Prog Drug Res 2008; 66: 189-216.

9 Lin CL, Huang HC, Lin JK. Theaflavins attenuate hepatic lipid accumulation through activating AMPK in human HepG2 cells. J Lipid Res 2007; 48: 2334-43.

10 Zang M, Xu S, Maitland-Toolan KA, Zuccollo A, Hou X, Jiang B, et al. Polyphenols stimulate AMP-activated protein kinase, lower lipids, and inhibit accelerated atherosclerosis in diabetic LDL receptor-deficient mice. Diabetes 2006; 55: 2180-91.

11 Baur JA, Pearson KJ, Price NL, Jamieson HA, Lerin C, Kalra A, et al. Resveratrol improves health and survival of mice on a high-calorie diet. Nature 2006; 444: 337-42.

12 Yu X, McCorkle S, Wang M, Lee Y, Li J, Saha AK, et al. Leptinomimetic effects of the AMP kinase activator AICAR in leptin-resistant rats: prevention of diabetes and ectopic lipid deposition. Diabetologia 2004; 47: 2012-21.

13 Song Z, Deaciuc I, Zhou Z, Song M, Chen T, Hill D, et al. Involvement of AMP-activated protein kinase in beneficial effects of betaine on high-sucrose diet-induced hepatic steatosis. Am J Physiol Gastrointest Liver Physiol 2007; 293: G894-G902.

14 Park KG, Min AK, Koh EH, Kim HS, Kim MO, Park HS, et al. Alphalipoic acid decreases hepatic lipogenesis through adenosine monophosphate-activated protein kinase (AMPK)-dependent and AMPKindependent pathways. Hepatology 2008; 48: 1477-86.

15 Yang J, Maika S, Craddock L, King JA, Liu ZM. Chronic activation of AMP-activated protein kinase-alpha1 in liver leads to decreased adiposity in mice. Biochem Biophys Res Commun 2008; 370: 24853.

16 Zhou G, Myers R, Li Y, Chen Y, Shen X, Fenyk-Melody J, et al. Role of AMP-activated protein kinase in mechanism of metformin action. J Clin Invest 2001; 108: 1167-74.

17 Bajaj M, Suraamornkul S, Pratipanawatr T, Hardies $\sqcup$, Pratipanawatr W, Glass L, et al. Pioglitazone reduces hepatic fat content and augments splanchnic glucose uptake in patients with type 2 diabetes. Diabetes 2003; 52: 1364-70.

18 Huang WH, Lee AR, Yang $\mathrm{CH}$. Antioxidative and anti-inflammatory activities of polyhydroxyflavonoids of Scutellaria baicalensis GEORGI. Biosci Biotechnol Biochem 2006; 70: 2371-80.

19 Waisundara VY, Hsu A, Huang D, Tan BK. Scutellaria baicalensis enhances the anti-diabetic activity of metformin in streptozotocininduced diabetic Wistar rats. Am J Chin Med 2008; 36: 517-40.

20 Regulska-llow B, Biernat J, Grajeta H, llow R, Drzewicka M. Influence of bioflavonoids from the radix extract of Scutellaria baicalensis on the level of serum lipids, and the development of laboratory rats fed with fresh and oxidized fats. Nahrung 2004; 48: 123-8.

21 Sekiya N, Kogure T, Kita T, Kasahara Y, Sakakibara I, Goto H, et al. Reduction of plasma triglyceride level and enhancement of plasma albumin concentration by Oren-gedoku-to administration. Phytomedicine 2002; 9: 455-60.

22 Sekiya N, Kainuma M, Hikiami H, Nakagawa T, Kouta K, Shibahara $\mathrm{N}$, et al. Oren-gedoku-to and Keishi-bukuryo-gan-ryo inhibit the progression of atherosclerosis in diet-induced hypercholesterolemic rabbits. Biol Pharm Bull 2005; 28: 294-8.

23 Fujimoto M, Tsuneyama K, Kainuma M, Sekiya N, Goto H, Takano Y, et al. Evidence-based efficacy of Kampo formulas in a model of non alcoholic fatty liver. Exp Biol Med (Maywood) 2008; 233: 328-37.

24 Heider JG, Boyett RL. The picomole determination of free and total cholesterol in cells in culture. J Lipid Res 1978; 19: 514-8.

25 Hardie DG. AMP-activated/SNF1 protein kinases: conserved guardians of cellular energy. Nat Rev Mol Cell Biol 2007; 8: 774-85.

26 Caldwell SH, Argo CK, Al-Osaimi AM. Therapy of NAFLD: insulin sensitizing agents. J Clin Gastroenterol 2006; 40 Suppl 1: S61-S66.

27 Shimano H, Yahagi N, memiya-Kudo M, Hasty AH, Osuga J, Tamura $\mathrm{Y}$, et al. Sterol regulatory element-binding protein-1 as a key transcription factor for nutritional induction of lipogenic enzyme genes. J Biol Chem 1999; 274: 35832-9.

28 Zang M, Zuccollo A, Hou X, Nagata D, Walsh K, Herscovitz H, et al. AMP-activated protein kinase is required for the lipid-lowering effect of metformin in insulin-resistant human HepG2 cells. J Biol Chem 2004; 279: 47898-905.

29 Chang CP, Huang WT, Cheng BC, Hsu CC, Lin MT. The flavonoid baicalin protects against cerebrovascular dysfunction and brain inflammation in experimental heatstroke. Neuropharmacology 2007; 52: 1024-33.

30 Kim DH, Kim HK, Park S, Kim JY, Zou Y, Cho KH, et al. Short-term feeding of baicalin inhibits age-associated NF-kappaB activation. Mech Ageing Dev 2006; 127: 719-25.

31 Stickel F, Schuppan D. Herbal medicine in the treatment of liver diseases. Dig Liver Dis 2007; 39: 293-304.

32 Viollet B, Foretz M, Guigas B, Horman S, Dentin R, Bertrand L, et al. Activation of AMP-activated protein kinase in the liver: a new strategy for the management of metabolic hepatic disorders. J Physiol 2006; 574: 41-53.

33 Misra P. AMP activated protein kinase: a next generation target for total metabolic control. Expert Opin Ther Targets 2008; 12: 91-100.

34 Hardie DG. The AMP-activated protein kinase pathway--new players upstream and downstream. J Cell Sci 2004; 117: 5479-87.

35 Ma KL, Ruan XZ, Powis SH, Chen Y, Moorhead JF, Varghese Z. Inflammatory stress exacerbates lipid accumulation in hepatic cells and fatty livers of apolipoprotein E knockout mice. Hepatology 2008; 48: 770-81.

36 Steinberg GR. Inflammation in obesity is the common link between defects in fatty acid metabolism and insulin resistance. Cell Cycle 2007; 6: 888-94.

37 Wan JY, Gong X, Zhang L, Li HZ, Zhou YF, Zhou QX. Protective effect of baicalin against lipopolysaccharide/ $D$-galactosamine-induced liver injury in mice by up-regulation of heme oxygenase-1. Eur J Pharmacol 2008; 587: 302-8. 